\title{
Supervision de stage à distance à l'aide du numérique
}

\section{Collaboration entre superviseurs et acteurs du milieu scolaire pour l'accompagnement de stagiaires avec un lien d'emploi en enseignement au secondaire}

\author{
Matthieu Petit, Ph. D. \\ Professeur agrégé au département de pédagogie de la Faculté d'éducation \\ de l'Université de Sherbrooke \\ matthieu.petit@usherbrooke.ca
}

«Dans quelques années, il sera impossible de concevoir le travail de supervision de stage en enseignement sans l'utilisation d'outils technologiques.» (Vazquez et Sevillano, 2013, p. 85)

Lorsque la supervision de stage en enseignement se fait à distance, l'approche collaborative des acteurs du milieu universitaire et de ceux du milieu scolaire nécessite des ajustements, voire de nouvelles façons de faire au service d'un dialogue favorable au développement professionnel des stagiaires. Si cette collaboration relève à la fois de possibilités et de limites à l'égard de la « présence » lors d'une supervision de stage à distance, notre recherche en révèle certaines caractéristiques selon un contexte particulier. L'objet de cette recherche était initialement le sentiment de présence de stagiaires supervisés à distance; la collaboration entre les superviseurs et les acteurs du milieu scolaire à l'aide des technologies de l'information et de la communication (TIC) s'est imposée au cours de l'analyse des données et cet article y est consacré. Selon le cadre de l'apprentissage collaboratif à distance de Henri et Lundgren-Cayrol (2001), nous en viendrons à proposer un collectif d'accompagnement au sein duquel la collaboration comporte des interactions enseignantes, cognitives et sociales dans un environnement en ligne réunissant entre autres le stagiaire, le superviseur et les acteurs du milieu.

Mots-clés / Supervision, distance, numérique, présence, collaboration

\section{Superviser des stagiaires, malgré la distance}

Depuis plusieurs années, les recherches démontrent que l'accompagnement des stagiaires est d'une grande importance dans les programmes de formation conçus selon le modèle de l'alternance entre l'université et le milieu de pratique (Boutet et Rousseau, 2002; Ferrier-Kerr, 2009). En ce sens, le 
développement professionnel des stagiaires relève en partie de la supervision offerte (Boudreau, 2009). $\mathrm{Au}$ Québec, les stages en enseignement sont habituellement supervisés par des représentants de l'université (cumulant une formation de deuxième cycle ainsi qu'une expérience pertinente en accompagnement et en enseignement) qui se rendent à quelques reprises dans les milieux scolaires accueillant leurs stagiaires. Lors de ces visites, les superviseurs rencontrent les enseignants (nommés mentors, enseignants associés ou maîtres de stage) qui accueillent et guident les stagiaires de leur cohorte; ce sont les directions d'école qui autorisent certains enseignants d'expérience à recevoir des stagiaires. Chaque cohorte d'un superviseur peut compter environ une dizaine de personnes, voire davantage selon les contextes de stage. Se voulant favorables à la persévérance et à la réussite des étudiants, ces petits regroupements peuvent constituer ce que Tinto (2003) nomme des communautés d'apprentissage. Lors des stages, les membres de ces regroupements sont séparés, répartis dans différentes écoles d'un territoire, chacun se retrouvant dans son milieu de pratique.

Les stages donnent souvent l'occasion aux étudiants de retourner dans leur région d'origine ou d'explorer d'autres réalités par des stages à l'étranger. Conséquemment, le coût des déplacements des superviseurs de stage représente une dépense importante dans le budget de fonctionnement des programmes (Grable, Hunt et Unrue, 2008) et la fatigue des superviseurs causée par les grandes distances à parcourir peut nuire à la qualité de l'accompagnement des stagiaires (Nault et Nault, 2001). Ainsi, l'étalement sur le territoire des personnes à former et la mobilité étudiante à l'échelle internationale - ainsi que les défis à surmonter dans le placement traditionnel des stagiaires (Gronn, Romeo, McNamara et Teo, 2013) - ont amené certaines universités à adopter différentes mesures de formation à distance (FAD) pour la supervision des stages, et ce non seulement en éducation mais dans de nombreux domaines en sciences humaines ou en sciences de la santé (Petit, Dionne et Brouillette, 2017).

\section{Être présent, à distance}

La distance implique parfois que certains stages se font sans supervision directe (i.e. sans le présentiel), mais paradoxalement, la distance peut rapprocher (Caraguel, 2012) puisqu'il devient alors incontournable de créer de la présence à distance en e-learning (Jézégou, 2010). Pour Chipchase, Hill, Dunwoodie, Allen, Kane, Piper et Russell (2014), la supervision de stage à distance (télésupervision ou e-supervision) se définit par un processus de supervision avec un formateur à distance utilisant des TIC. Les objectifs de la supervision de stage à distance demeurent les mêmes qu'en présentiel, mais le rôle des superviseurs change (Hamel, 2012). Les superviseurs jouent leur nouveau rôle entre autres par le « design » du nouvel encadrement qu'ils proposent (Jiang, Parent et Eastmond, 2006). Pour Arbaugh (2010), il est important que ce design - qui relève de l'organisation, de la pertinence des travaux proposés et de la clarté des intentions pédagogiques (Hosler et Arend, 2012) - soit fait avant le déroulement de l'activité. Par exemple, certaines consignes peuvent guider une bonne utilisation de la vidéo en stage afin de remplacer les traditionnelles visites de supervision lors desquelles les superviseurs font habituellement l'essentiel de l'évaluation des stagiaires de leur cohorte.

L'arrivée massive des TIC a ouvert la porte à une approche multidimensionnelle pour la supervision de stage à distance (Pellerin, 2010). Dans ce contexte, plusieurs évidences (ou traces) du développement professionnel des stagiaires doivent être soumises aux superviseurs, ce qui n'est pas nécessairement le cas lors d'une supervision en présentiel (Alger et Kopcha, 2009). En effet, les TIC (vidéos synchrones ou asynchrones, services de partage de fichiers lourds, visioconférence, forums électroniques, blogues, portfolio électronique, etc.) permettent un accompagnement en ligne, voire une évaluation d'une grande richesse (Conn, Roberts et Powell, 2009). Par exemple, la supervision a plus d'impact si elle se déroule tôt dans le stage, en plus d'être fréquente (Orr, 2010). Grâce aux TIC et au mode asynchrone de la FAD,

revue-mediations.teluq.ca | vol. 01 , no. 1 
les superviseurs ne sont plus autant limités par les enjeux relevant de la distance à parcourir et du calendrier quant au moment et à la fréquence. Pour tout superviseur, visiter en début de stage plus d'une dizaine d'étudiants dans des lieux différents peut s'avérer un véritable casse-tête. En ce sens, Schwartz-Bechet (2014) souligne que la supervision de stage à distance permet de dégager plus de temps pour observer, analyser et discuter, tout en donnant de nouvelles opportunités de réflexion pour les stagiaires (entre autres grâce aux éléments soumis aux superviseurs). Ainsi, au-delà des raisons économiques - la e-supervision permettant de limiter les coûts principalement liés aux déplacements des superviseurs (Joseph et Brennan, 2013) -, il peut donc y avoir une plus-value pédagogique à la supervision de stage à distance.

Par contre, la rétention des étudiants en FAD représente un enjeu majeur (Traver, Volchok, Bidjerano et Shea, 2014). Afin d'éviter l'abandon des stagiaires qui vivent de l'isolement, il faut veiller à la qualité de l'accompagnement en supervision de stage à distance. À la fois pédagogique, organisationnel, technologique et éthique (Petit, 2015), le design des dispositifs numériques doit ainsi considérer le sentiment de présence des stagiaires. La distance peut être perçue comme un espace d'interactions, mesurable par différents types de présence. Le modèle de la communauté d'apprentissage en ligne (CAL) de Garrison, Anderson et Archer (2000) qui s'impose en recherche depuis plus de 10 ans (Deaudelin, Petit et Brouillette, 2016) en compte trois : présence enseignante (PE), présence cognitive $(P C)$ et présence sociale (PS) (voir figure 1). Selon une posture socioconstructiviste favorable aux apprentissages en enseignement supérieur (Akyol et Garrison, 2011), les trois types de présence de la CAL donnent lieu à une expérience éducative à la fois riche et significative (Kozan et Richardson, 2014). La PE comporte trois dimensions : le design et l'organisation, la facilitation des échanges et l'enseignement. La PC en comprend quatre: l'évocation d'éléments déclencheurs, l'exploration de solutions, l'intégration et la résolution du problème posé. Quant à la PS, elle s'opérationnalise à travers l'expression de l'affectivité, la communication ouverte et la cohésion du groupe réunissant autant les apprenants que les formateurs.

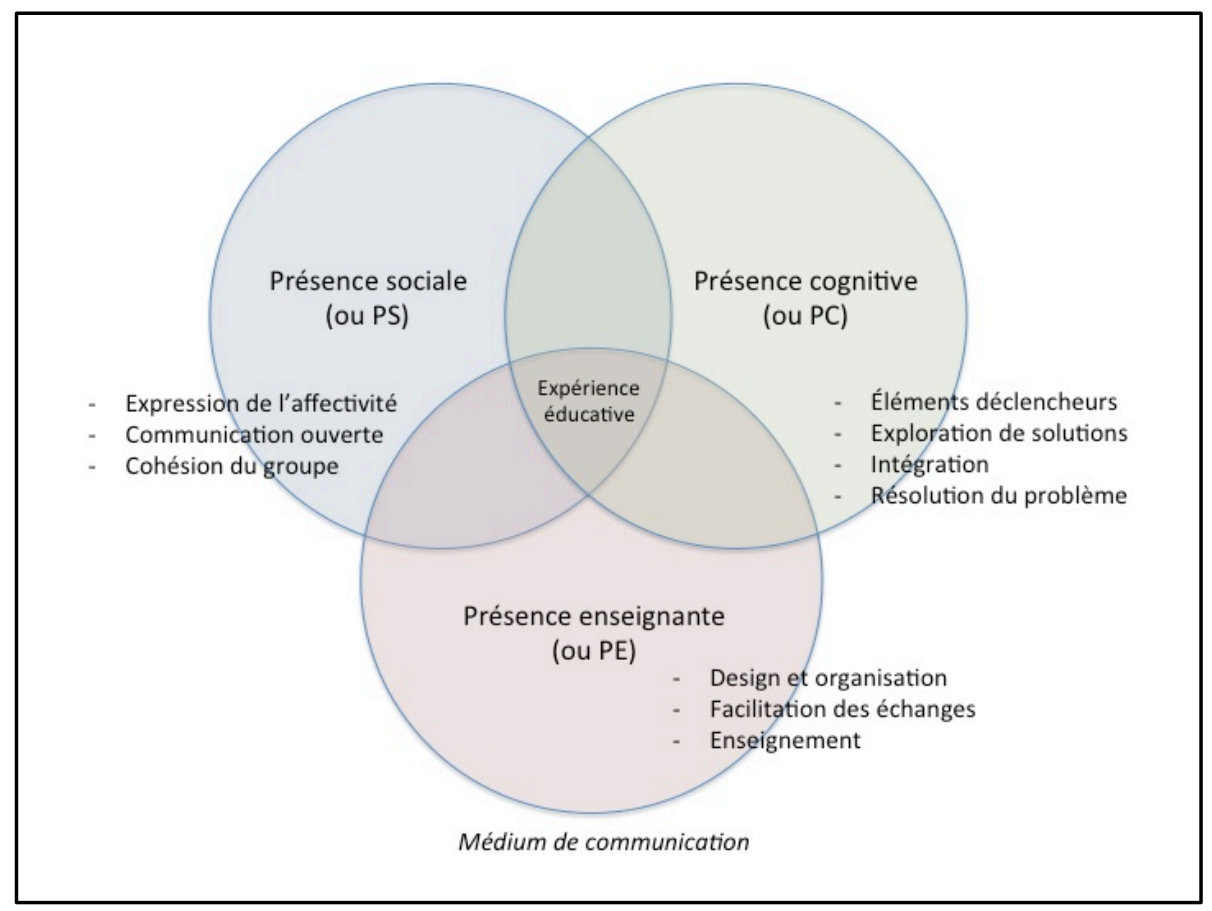

Figure 1. Modèle théorique de la communauté d'apprentissage en ligne (Garrison et al., 2000) 
Le modèle de la CAL permet d'aborder diverses expériences éducatives en ligne, dont celle relevant d'une formation pratique supervisée à l'aide des TIC. Le contexte de supervision de stage à distance qui nous intéresse dans cet article présente certaines particularités que nous abordons dans la prochaine section.

\section{Accompagner des stagiaires avec un lien d'emploi}

Même si la supervision de stage à distance s'impose dans un nombre grandissant de programmes de différents domaines (Petit, Dionne et Brouillette, 2017), nous ne savons que très peu de choses sur les pratiques des superviseurs de ces programmes, dont ceux en enseignement. Dans le cadre de nos recherches, nous nous sommes intéressés au cas d'un programme de formation à l'enseignement offert uniquement en ligne - la maîtrise qualifiante en enseignement au secondaire (MQES) de l'Université de Sherbrooke - qui comporte deux stages supervisés à distance. Ainsi, les superviseurs de ce programme exercent leurs compétences (Portelance, Gervais, Lessard et Beaulieu, 2008) sans se rendre physiquement dans les milieux scolaires où œuvrent les stagiaires, à moins que ceux-ci éprouvent des difficultés majeures dans un contexte particulier et qu'un échec soit envisagé pour le stage.

Comme le soulignent Carlisle, Carlisle, Hill, Kirk-Jenkins et Polychronopoulos (2013), une supervision en présentiel ne convient habituellement pas à des étudiants habitués à la flexibilité de la FAD qui permet à plusieurs d'entre eux d'occuper un emploi souvent à temps plein. C'est tout particulièrement le cas pour la MQES car elle accueille principalement du personnel enseignant au secondaire - avec une formation disciplinaire pertinente, mais habituellement sans diplôme universitaire en éducation - qui suit cette formation de deuxième cycle en ligne afin d'acquérir le brevet d'enseignement pour le Québec tout en continuant de travailler (parfois avec un «100\% de tâche»). II s'agit donc de stagiaires avec un lien d'emploi auprès d'une commission scolaire ou d'une institution privée d'enseignement qui ont besoin du brevet pour s'assurer d'une certaine stabilité d'emploi, voire d'une éventuelle permanence.

Actuellement, une seule rencontre en présentiel permet à chaque superviseur de la MQES de réunir sa cohorte en début de session (à l'université ou dans un lieu stratégique sur le territoire québécois) et de démarrer l'accompagnement de ses stagiaires répartis un peu partout en province. Par cette introduction en présentiel lors du premier stage (ainsi qu'une présentation devant jury à la fin du deuxième stage), nous pouvons qualifier cette supervision d'hybride (Conn et al., 2009).

Afin d'atteindre les objectifs de sa formation pratique en enseignement (MÉLS, 2008), le stagiaire prend habituellement en charge certains (voire tous les) groupes de son enseignant associé qui demeure le responsable du bon déroulement des cours et de l'apprentissage de ses élèves. À la MQES, la situation est différente. Puisque les stagiaires sont pour la plupart des enseignants avec un lien d'emploi, le représentant du milieu scolaire n'est pas un enseignant associé, mais il est désigné comme étant le mentor du stagiaire. II s'agit habituellement d'un collègue du stagiaire, avec une grande expérience dans la discipline concernée. Le mentor ne partage pas ses groupes d'élèves avec le stagiaire car celui-ci a habituellement déjà les siens. L'accompagnement par le mentor se fait donc en parallèle de sa tâche habituelle. On ne peut exiger du mentor, par exemple, qu'il aille observer son stagiaire lorsqu'il enseigne. La présence des mentors dans la classe des stagiaires de la MQES est donc limitée, voire parfois rare si on la compare à celle des enseignants associés d'un programme de premier cycle. Cela peut poser problème lorsqu'un stagiaire vit des difficultés, mais heureusement, si le mentor n'est pas disponible, un membre de la direction d'école risque peut-être de l'être.

La supervision pédagogique des jeunes enseignants faisant partie du rôle des directions d'école, les différents membres d'une direction (que ce soit la direction principale ou les directions adjointes) sont 
amenés à intervenir auprès des stagiaires de la MQES, ce qui n'est pas toujours le cas des programmes plus traditionnels en enseignement. Ainsi, la collaboration des superviseurs de la MQES avec les milieux de pratique implique non pas une, mais au moins deux personnes-ressources: le mentor et la direction d'école. Selon Moussay, Étienne et Méard (2009), cet accès à plusieurs accompagnateurs - dont la direction d'école (Moussay, 2007) - est favorable au développement professionnel du stagiaire. À la MQES, même si la complémentarité entre le mentor et le membre de la direction diffère d'une école à l'autre, les deux doivent remplir et co-signer le cahier d'évaluation sommative du stagiaire.

Nos recherches ont permis d'établir le dispositif pédagogique et numérique utilisé par les superviseurs de la MQES (Petit, 2016). La perception de présence des stagiaires a également été mesurée. À l'aide d'un questionnaire électronique découlant du modèle de la CAL (Garrison, Cleveland-Innes et Fung, 2010), il ressort que, généralement, les stagiaires s'accordent sur le fait que la supervision de stage à distance à la MQES permet la création d'un sentiment de présence (Petit, 2016). De plus, la perception des stagiaires est majoritairement favorable autant pour la PE que pour la PC et la PS (Ibid.). Parmi les conditions favorables à la création de présence au sein des cohortes de stagiaires, notons l'engagement du superviseur (entre autres par sa disponibilité et la régularité de ses interactions) ainsi que la relation de confiance qu'il établit avec ses stagiaires (Ibid.). Ces résultats permettent de croire que les pratiques de la MQES réunissent plusieurs conditions favorables à la création d'un sentiment de présence chez les étudiants par la supervision de stage à distance, et que celle-ci peut constituer une véritable alternative à la supervision traditionnelle.

\section{Questions, objectif et considérations méthodologiques}

Qu'en est-il des mentors et des membres de la direction qui accompagnent les stagiaires de la MQES dans les milieux scolaires? Dans le contexte d'une supervision de stage à distance, ils peuvent eux aussi se sentir isolés du fait de l'absence physique des superviseurs. Avec la distance, les superviseurs doivent redéfinir leur rôle afin de collaborer avec les personnes-ressources dans le contexte de la MQES. À moins de problématiques majeures, leur présence auprès des mentors et de la direction passe donc par une utilisation des TIC. En référence à la triade en supervision universitaire (Boutet et Rousseau, 2002), cette " présence virtuelle » permet-elle aux superviseurs de former des tétrades avec les stagiaires et les deux représentants du milieu scolaire (voir figure 2) ? À la lumière de ce questionnement et de l'étude du cas de la MQES, notre objectif est d'identifier des caractéristiques de la collaboration entre les personnes superviseures et les acteurs du milieu scolaire en enseignement au secondaire par un dispositif de supervision de stage à distance.

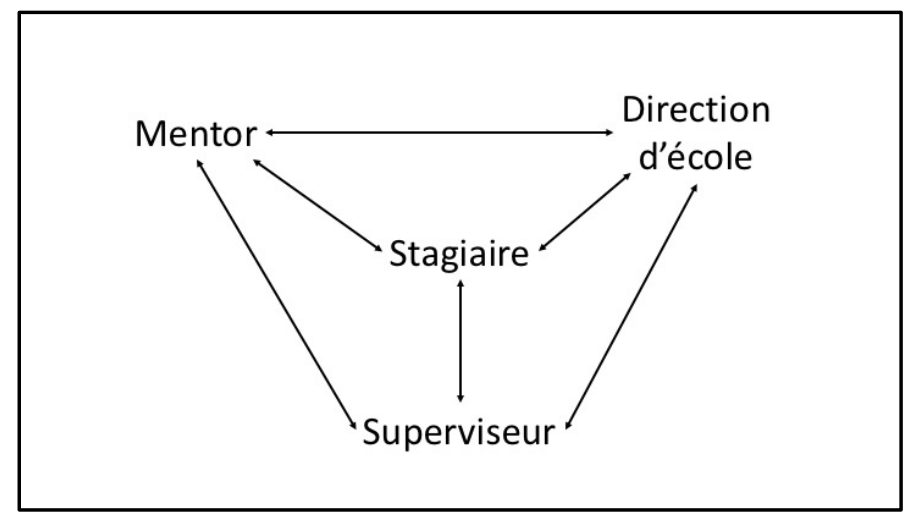

Figure 2. Tétrade pour l'accompagnement à distance des stagiaires à la MQES 
Le concept de collaboration s'est imposé dans le cadre d'une étude exploratoire-descriptive. Une série d'entretiens individuels semi-structurés ont eu lieu auprès de cinq superviseurs de la MQES (un échantillon de convenance) afin d'aborder les possibilités et les limites en termes de présence d'une supervision de stage à distance. Le questionnaire (composé de questions ouvertes) était inspiré du modèle de Lehman et Conceição (2010).

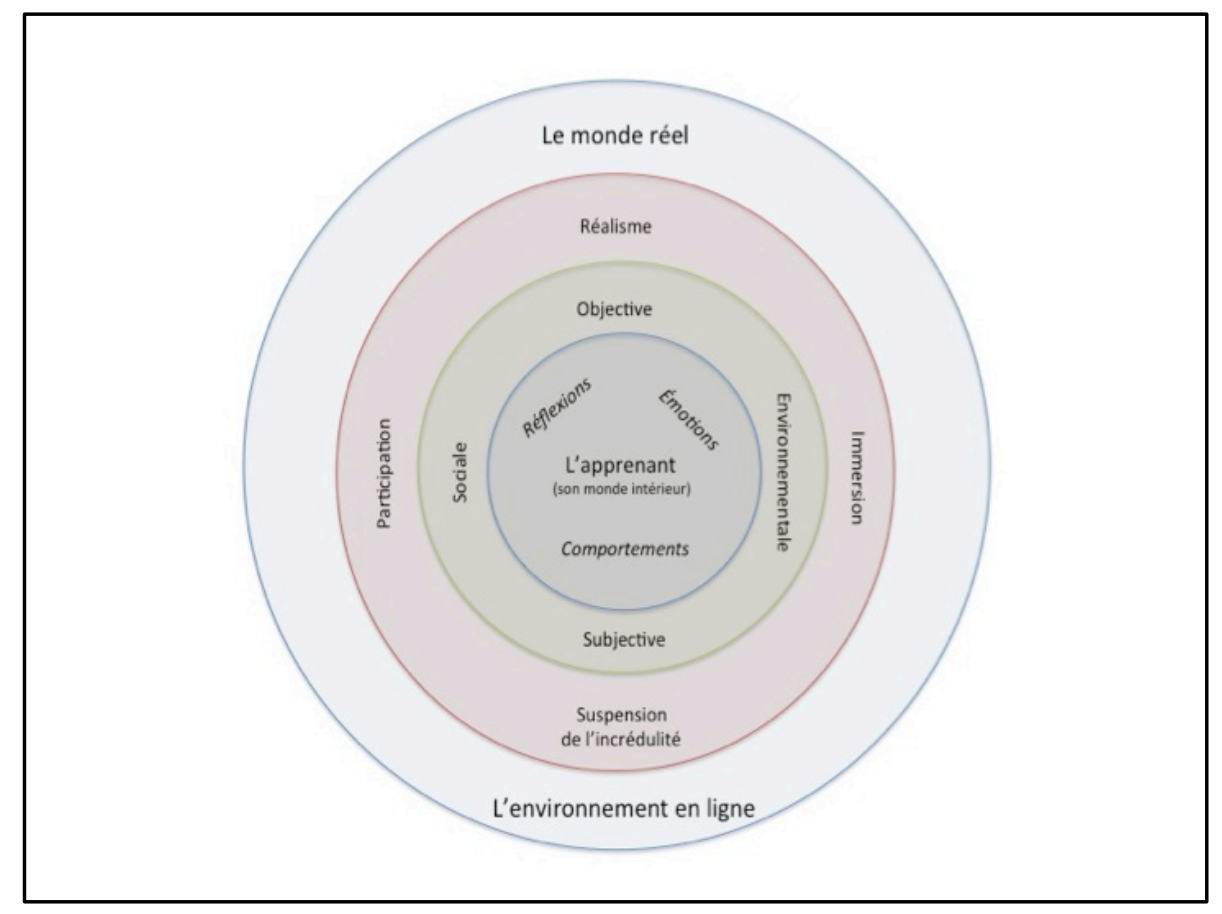

Figure 3. Modèle pour la création du sentiment de présence en ligne (Lehman et Conceição, 2010).

Mettant en commun plusieurs dimensions de travaux sur le sentiment de présence - dont ceux de Garrison et al. (2000) -, ce modèle pour la FAD considère non seulement les types d'expérience en ligne et les modes de présence, mais il mène également à six facteurs de présence qui ont constitués les catégories initiales de notre codage du verbatim des entrevues : 1- le type et la convergence du contenu, 2- le format de l'expérience d'apprentissage, 3- les stratégies d'interaction, 4- le rôle du formateur (ou du superviseur), 5- le type de technologie, et 6- les sortes de support fourni. Selon Lehman et Conceição (2010), ces facteurs peuvent guider le formateur quant au design et au déroulement d'une formation à distance - dont une supervision de stage à distance - favorisant la création d'un sentiment de présence.

Le codage selon des unités de sens étant ouvert aux catégories émergentes (autres que celles des facteurs de présence), l'analyse a révélé des résultats concernant la collaboration des superviseurs avec les différentes personnes-ressources du milieu scolaire. Une validation interjuge a permis d'assurer une justesse quant à la signification et à la compréhension des catégories (initiales et émergentes). Les résultats relèvent de la perception des superviseurs sollicités à l'égard de cette collaboration à distance. Sans pouvoir être généralisés, ces résultats permettent de dégager certaines pratiques de supervision de stage à distance de ce programme atypique.

Dans la prochaine section, nous nous attardons donc sur les caractéristiques du dispositif collaboratif réunissant les personnes superviseures et les acteurs du milieu scolaire en enseignement au secondaire 
lors d'une supervision de stage à distance. Ces résultats s'inscrivent en complémentarité aux possibilités et limites en termes de présence d'une supervision de stage à distance (Petit, 2016). Dans les extraits du verbatim des entretiens (qui se sont faits dans le respect des normes éthiques en vigueur), les noms des superviseurs ont été remplacés par S1, S2, S3, S4 et S5 afin d'assurer leur anonymat.

\title{
Résultats : collaboration avec les milieux scolaires, selon les superviseurs de la MQES
}

\author{
Installer un climat de confiance, même à distance
}

Pour les superviseurs de la MQES, il s'avère tout particulièrement important d'établir un lien de confiance, voire leur présence, auprès des acteurs du milieu scolaire. Que ce soit par téléphone ou courriel, il est du rôle des superviseurs d'effectuer un premier contact avec les mentors et les directions d'école avant le début du stage afin d'établir les bases de la collaboration : "Cette conversation est très importante. Je leur envoie à ce moment la grille d'évaluation. " (S3) Cela permet également aux superviseurs d'inviter les personnes-ressources du milieu à bien parcourir les documents de stage qui précisent entre autres les rôles de chaque membre de la tétrade.

Puisque les superviseurs ne sont pas amenés à mettre les pieds dans les écoles, cette relation est cruciale pour eux. À certains égards, ce sont les mentors qu'ils perçoivent comme les pivots des milieux scolaires :

S'il y a des problèmes avec l'outil, avec l'utilisation de la caméra, je vais devoir me fier un peu plus aux commentaires du mentor. Mais je dois aussi discuter avec eux pour connaître leur vision, leurs attentes... Parfois ils peuvent sentir qu'ils sont eux aussi évalués. II faut mettre ça au clair, les tenir au courant, établir une relation de confiance afin que je sois informé des situations qui se déroulent en classe. (S1)

Ainsi, malgré la distance, les superviseurs peuvent bénéficier de la réelle présence des mentors sur les lieux du stage, voire parfois dans la classe. Les problèmes technologiques pouvant être chronophages, l'aide des mentors à cet égard est assurément appréciée. Et en raison de la nécessité qu'ont les superviseurs d'établir un bon premier contact, certaines craintes des mentors (qu'on retrouve également chez les enseignants associés lors de stages plus traditionnels) peuvent être abordées pour ensuite être atténuées ou disparaître pour la durée du stage.

\section{Encourager l'engagement des mentors}

Au cours du stage, la communication entre le superviseur et le mentor passe entre autres par le stagiaire (et l'utilisation qu'il fait des TIC) :

Je demande au mentor d'être présent pour le retour dans la vidéo. Les deux peuvent jaser devant la caméra et dire ce qu'ils pensent du cours. J'essaie de trouver des moyens à distance qui font que je suis en contact réel avec le monde, ce que j'ai en présence, mais pas nécessairement en ligne, ou de la même façon. (S3) 
Qu'en est-il si le mentor enseigne en même temps que le stagiaire? "Même si le mentor n'a pas vu la classe, je veux qu'il soit là pour échanger parce qu'il connaît le stagiaire. Je veux qu'ils réfléchissent ensemble comme lors d'un entretien post-leçon. » (S2)

Quant à la posture des mentors, les superviseurs font preuve d'ouverture, tant et aussi longtemps que les mentors sont disponibles, investis : "Les échos que j'ai à cet égard, c'est que les mentors sont très présents. II faut que ce soit une présence régulière. La mentalité importe peu, mais il faut un suivi constant entre le mentor et l'étudiant, qu'ils travaillent de pair » (S2). On suppose qu'un accompagnement collégial est facilité par le contexte de la MQES : le stagiaire et le mentor sont des collègues au quotidien.

\section{Impliquer les directions d'établissements}

Si la connivence entre un stagiaire et son mentor peut être un atout pour l'accompagnement selon une structure de coopération (St-Arnaud, 2003), elle est pourtant perçue comme étant problématique par les superviseurs du programme. "Je ne peux pas demander à un pair d'évaluer un collègue sans être en conflit d'intérêt. " (S2) Quand vient le temps d'évaluer la personne stagiaire, ça peut laisser sousentendre une certaine partialité. C'est pourquoi les directions d'écoles sont invitées à prendre part à l'évaluation des stagiaires de la MQES qui, rappelons-le, sont des enseignants à l'emploi de leur école. « Normalement, c'est la direction qui fait l'évaluation. [...] Lorsque le stagiaire n'est pas en difficulté, ce n'est pas risqué de faire intervenir le mentor davantage, mais on ne veut pas que le mentor sanctionne un échec parce que c'est un collègue." (S4) Ce superviseur a cette suggestion pour les directions d'école : "Je leur suggère fortement d'aller voir leurs employés en classe. Pour des cas d'exception, c'est le mentor qui évalue, mais je dis à la direction qu'elle va devoir signer l'évaluation » (S2).

Aux yeux des superviseurs de la MQES, la collaboration est donc plus importante avec la direction d'école. " Je dis au directeur que je compte sur lui. II peut aller dans la classe et je l'invite à le faire au moins une fois, ou à regarder les vidéos. Si le directeur est en confiance avec nous, avec l'université, lui aussi peut signaler un problème. Le mentor, c'est plutôt rare que je me fie sur lui. » (S3) Pour cet autre superviseur, le déséquilibre est clair entre les mentors et les membres de la direction quand vient le temps d'entretenir la collaboration: " Je contacte très peu les mentors » (S2). Et la collaboration avec la direction prend tout son sens si le stagiaire fait face à la possibilité d'un échec:

Si je me questionne sur les compétences d'un stagiaire, je me lie à la direction de l'école, et on travaille ensemble. Si l'étudiant est possiblement en situation d'échec, c'est certain que je vais me déplacer. C'est ce qu'on a convenu. Quitte à y aller plus d'une fois. Chose certaine, les téléphones se font à une plus grande fréquence à ce moment-là. (S2)

\section{Prévenir les conflits ainsi que l'isolement des stagiaires}

Cette nouvelle dynamique sur les lieux de stage, soit celle impliquant le stagiaire, le mentor et la direction d'école, n'est malheureusement pas toujours au service de l'accompagnement des stagiaires :

Il y a parfois des dynamiques avec le mentor et la direction qui fait en sorte que le stagiaire se ramasse seul. J'ai parfois senti des problèmes qui relevaient plus de la gestion de l'école et de l'équipe que de la gestion de classe. Quand le mentor a de la difficulté, imagine le stagiaire s'il n'est pas épaulé par la direction d'école. À ce moment-là, tu gères des relations interpersonnelles et interprofessionnelles, et ça devient délicat. C'est un rôle qu'on doit jouer. 
Au sein de cette tétrade, le superviseur joue donc parfois le rôle ingrat d'arbitre, mais à cet égard, le principal nouveau rôle des superviseurs en contexte de supervision de stage à distance relève d'une prévention accrue des conflits ou de tout autre type de dérapage :

Je dis [aux stagiaires] que s'ils ont un conflit avec la direction ou avec le mentor, [qu'ils m'en parlent] au début du stage parce que vers la fin, il y a moins de chance que ça se règle. Pour moi, c'est facile de discuter avec une direction d'école. Mon but n'est pas de prendre pour l'un ou pour l'autre dans un conflit. Ici, c'est comment on sort de ça, comment on trouve une solution. C'est une façon pour moi de compenser le présentiel. J'essaie de trouver des indices...

Pour les superviseurs de la MQES, cette volonté de "compenser le présentiel " passe donc par une valorisation du rôle des personnes-ressources du milieu scolaire - tout particulièrement pour les directions d'établissement -, mais aussi par une vigilance aux signes d'isolement que peuvent manifester le stagiaire ou l'un des représentants du milieu scolaire. Notons qu'au-delà du téléphone et du courriel, ces superviseurs misent très peu sur d'autres TIC afin de témoigner de leur présence et de maintenir cette collaboration.

Dans la prochaine section, notre discussion autour de ces résultats se fait entre autres grâce aux écrits d'une recension systématique sur la supervision de stage à distance dans les différents domaines de formation postsecondaire (Petit, Dionne et Brouillette, 2017). Cela nous permet d'ouvrir la porte à une réflexion plus large quant à la collaboration afin de constituer un véritable collectif dans le contexte de la supervision de stage à distance. D'autres perspectives (dont celle de la formation des accompagnateurs) à l'égard de cette pratique novatrice étayent également notre réflexion.

\section{Discussion et perspectives : collaboration en sein d'un collectif d'accompagnement}

Dans le programme de supervision de stage à distance de la MQES, la collaboration entre les superviseurs et les personnes-ressources des milieux scolaires semble caractérisée par une volonté de prévenir les conflits et la nécessité d'une relation de confiance. Ce portrait tiré d'entretiens auprès de superviseurs expérimentés de la MQES témoigne également d'une faible utilisation des TIC afin de collaborer avec le milieu.

Orr (2010) souligne que la relation de confiance avec les formateurs du terrain demande plus de temps à établir à distance, et que la résistance est plus difficile à percevoir. Ainsi, cette condition gagnante pour la supervision en présentiel devient un incontournable pour la supervision de stage à distance, et les superviseurs doivent s'y prendre le plus tôt possible en plus de demeurer vigilants. Pour Cicco (2014), il est de la responsabilité des superviseurs de planifier une rencontre à distance avec les formateurs du terrain ainsi qu'avec le stagiaire pour réviser les rôles et responsabilités de chacun.

Mais est-ce que la confiance règne vraiment à la MQES ? Les superviseurs veulent que les mentors accompagnent les stagiaires, qu'ils soient présents, mais pas nécessairement qu'ils évaluent. Le jugement des mentors étant possiblement biaisé, ils placent l'évaluation entre les mains des membres des directions d'école (en posture d'autorité envers leurs enseignants, et parfois en conflit avec eux) sans nécessairement s'attendre à ce qu'ils s'investissent véritablement dans l'accompagnement des stagiaires. Même s'il est parfois ardu d'arrimer accompagnement et évaluation sommative, cette répartition des responsabilités nous apparaît inopportune. Alors que la triade composée du stagiaire, de 
l'enseignant associé et du superviseur est parfois critiquée (Hastings et Squires, 2002) - Rodgers et Keil (2007) la soupçonnent d'avoir parfois peu d'impact sur le développement professionnel du stagiaire -, la tétrade formée par le stagiaire, le superviseur, le mentor et la direction d'école peut sembler innovante, mais pas nécessairement avec la formule actuelle.

Au-delà des outils numériques et des nouveaux rôles pour les accompagnateurs dans un contexte de la supervision de stage à distance, un véritable changement de posture et de valeurs apparaît incontournable afin de bien «compenser le présentiel» et d'établir de bonnes conditions pour la collaboration. II faut repenser la relation dyadique accompagnateur-stagiaire selon une conception collective, à l'instar du modèle de présence en FAD qui relève d'une posture socioconstructiviste. Awaya, Mc Ewan, Heyler, Linsky, Lum et Wakukawa (2003) proposent de cesser les relations dissymétriques dans lesquelles les accompagnateurs se situent en surplomb du stagiaire, et d'opter pour des rapports de réciprocité, de partage d'expertise. Par exemple, il faut échapper au strict rituel d'entretiens post-leçon au cours duquel les accompagnateurs transmettent une longue liste de feedback au stagiaire (Loughran, 2002). Baecher, McCormack et Kung (2014) indiquent que les superviseurs monopolisent trop souvent le temps de parole (malgré leurs bonnes intentions). À cet égard, la solution implique de former les accompagnateurs, mais la patience est de mise car on ne change pas sa posture d'accompagnateur du jour au lendemain. II faut s'attendre à de la résistance.

Les mêmes rapports de réciprocité et de coopération doivent s'établir entre le superviseur et les accompagnateurs du milieu, mais également entre le mentor et la direction d'établissement dans le cas de la MQES. Avec cette posture, l'implication de la direction est la bienvenue. Lorsque le stagiaire peut avoir le soutien d'un collectif pour réfléchir sur ses compétences professionnelles, l'accompagnement joue alors davantage un rôle de catalyseur que de guidance (Moussay et al., 2009). Pour Le Cornu et Ewing (2008), il faut donc opter pour des modèles de supervision davantage centrés sur des pratiques d'apprentissages collaboratifs. Selon Henri et Lundgren-Cayrol (2001), l'étudiant est le principal agent de son propre développement au sein d'un groupe d'apprentissage collaboratif. II se doit d'y être actif, réflexif, autonome et motivé par une cible d'apprentissage à la fois personnelle et collective qu'il vise à atteindre en développant et en maintenant une saine interdépendance. À la fois complexe et dynamique, ce groupe s'apparente à une communauté d'apprentissage (Tinto, 2003). Ainsi, par de saines collaborations entre accompagnateurs, la tétrade de la MQES peut constituer une communauté d'apprentissage au service du développement professionnel du stagiaire. Un fort sentiment de communauté entre stagiaire, superviseur et formateurs du terrain peut contribuer à une expérience d'esupervision qui se révèle positive (Alger et Kopcha, 2011).

Qu'en est-il de la faible utilisation des TIC par les superviseurs de la MQES afin de collaborer avec les acteurs du milieu ? II faut former les superviseurs à cet égard (qui pourront ensuite mieux collaborer à distance avec le milieu scolaire). Mais par où commencer? Gronn et al. (2013) soulignent qu'une conscience préalable des exigences de la technologie serait bénéfique aux superviseurs. Et pour avoir des stagiaires réflexifs, il faut aussi des superviseurs réflexifs (Andrew, 2007). Ils sont alors en meilleure posture pour déterminer les actions à prendre quant aux TIC à leur disposition (Baecher et al., 2014).

Que ce soit en présentiel ou à distance, la culture de l'oral est très présente dans les écoles quand vient le temps d'évaluer (de manière formative ou sommative) les stagiaires et cela représente un risque élevé que les commentaires écrits soient insuffisants, voire non transmis au superviseur (Alger et Kopcha, 2009). La prévention s'avère de mise, tout particulièrement à distance. Le défi est donc d'augmenter les interactions avec les accompagnateurs des milieux éloignés afin de les former eux aussi et qu'ils comprennent mieux leur rôle (Hamel, 2012). La solution passe inévitablement par les TIC d'autant plus que, par l'entremise des outils numériques, les accompagnateurs des milieux scolaires peuvent se sentir davantage en collaboration avec les superviseurs à distance qu'avec ceux en présentiel (Ibid.). Wilson 
(2006, dans Mabunda, 2013) note également chez les formateurs du terrain un meilleur sentiment d'appartenance à la communauté en supervision de stage à distance qu'à celle d'un modèle de supervision plus traditionnelle. À la MQES, les mentors ou les directions d'école ne font pas partie d'une telle communauté liée à la supervision de leur stagiaire. En fait, ce sont eux qui sont tout particulièrement isolés par la supervision de stage à distance.

Si on les implique dans le dispositif numérique de l'institution universitaire, les acteurs du terrain peuvent se former (sur un environnement numérique d'apprentissage comme Moodle, par exemple) en plus d'utiliser des informations partagées en ligne (sur un forum de discussion, un e-portfolio, un blogue...) pour savoir comment accompagner et savoir sur quoi intervenir (Alger et Kopcha, 2011). Ainsi, une bonne utilisation des TIC pour communiquer et collaborer avec les formateurs du terrain aura un impact sur les stagiaires (Vazquez et Sevillano, 2013).

Selon Moussay et al. (2009), les autres enseignants (ou les spécialistes) de l'école peuvent aussi avoir un impact sur les stagiaires. Ils sont à inclure au sein du groupe d'apprentissage collaboratif car les stagiaires échangent avec eux au quotidien. De plus, ce collectif d'accompagnement doit aussi faire une place aux pairs, surtout ceux avec lesquels les stagiaires échangent au quotidien (si l'école compte plus d'un stagiaire), mais aussi ceux de leur cohorte. Pour Wolf (2003), il faut inviter le réseau de pairs à s'investir dans un véritable travail d'équipe. À la MQES, il est rare que plusieurs stagiaires se retrouvent dans une même école ; les échanges entre pairs se font principalement sur les forums de discussion des différentes cohortes. Avec les pairs, l'accompagnement peut être plus rapide et parfois plus adéquat que celui offert par un superviseur (Yeh, Chang, Chiang, Drost, Spelliscy, Carter et Chang, 2008). Ces auteurs soulignent une réduction de l'anxiété et une augmentation de l'empathie au sein de groupes d'accompagnement entre pairs. Par ces échanges (sur un forum ou autres TIC), les stagiaires remarquent qu'ils sont devenus des modèles pour les autres (Alger et Kopcha, 2011). Ils peuvent donc réduire leur dépendance aux experts externes - ce qui rejoint le modèle de l'apprentissage collaboratif d'Henri et Lundgren-Cayrol (2001) - et développer des compétences d'accompagnement, ainsi qu'une conscience quant à leurs propres compétences (Yeh et al., 2008). En ce sens, Joseph et Brennan (2013) identifient l'accompagnement par les pairs comme une voie significative pour favoriser l'apprentissage des stagiaires.

Ainsi, nous considérons que la transformation souhaitable quant à la collaboration entre les superviseurs et les différents intervenants en contexte de supervision de stage à distance en enseignement doit s'inscrire dans un collectif d'accompagnement autour du stagiaire, et lorsque nous représentons celui-ci, nous obtenons la figure suivante : 


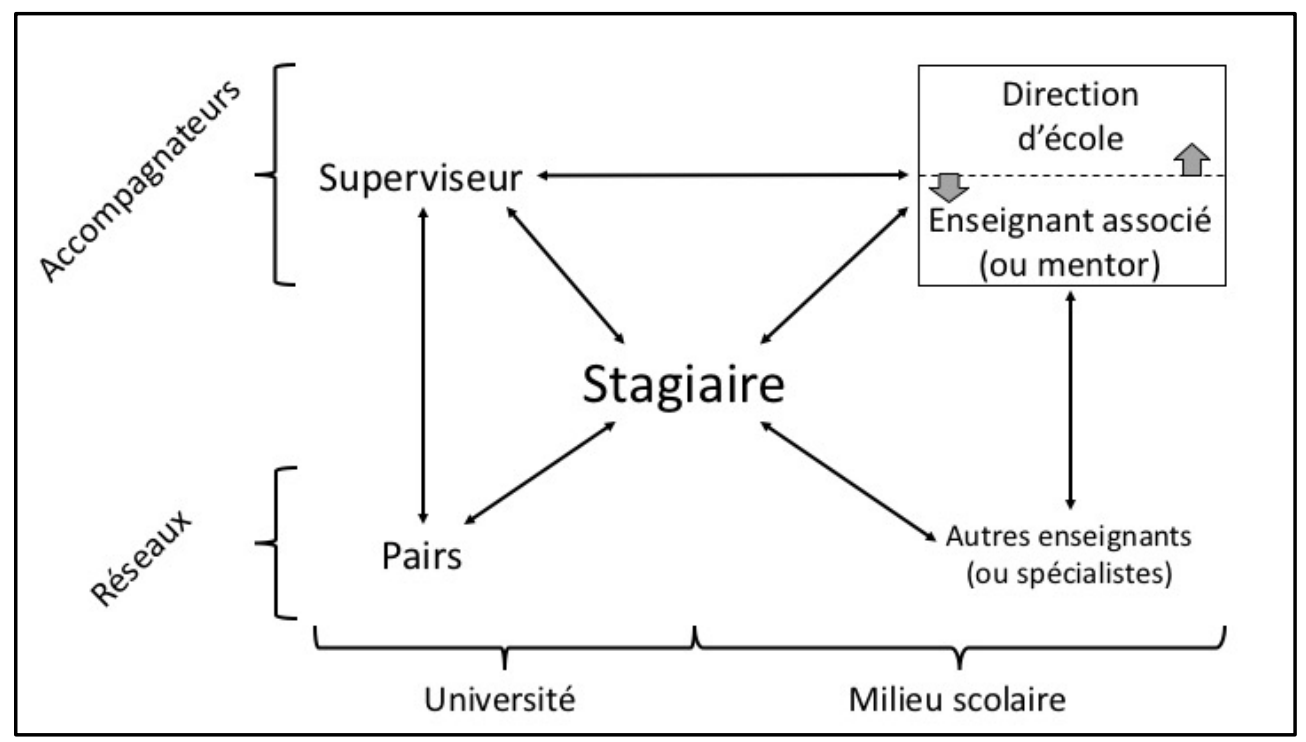

Figure 4. Collectif d'accompagnement du stagiaire en enseignement

Cette représentation témoigne bien de la complexité des relations et des dispositifs collaboratifs à mettre en place pour la supervision de stage à distance. Selon Peraya (2015), le transfert de cadres entre la formation en présentiel et la FAD peut se faire dans les deux sens, et ce à certaines conditions. Ainsi, ce collectif pour mettre en dialogue tous les acteurs de l'accompagnement des stagiaires de la MQES pourrait s'appliquer à une supervision de stage en présentiel.

\section{Conclusion}

Que ce soit pour des programmes de formation en éducation, médecine, travail social, psychologie, psychothérapie, orthophonie, ergothérapie, orientation ou un autre domaine, les chercheurs soulignent le grand potentiel de la supervision de stage à l'aide des TIC (Petit, Dionne et Brouillette, 2017). Au-delà de l'isolement des stagiaires en FAD, certains défis méritent notre vigilance. Rousmaniere, Abbass et Frederickson (2014) identifient des enjeux quant à la sécurité, la confidentialité, l'éthique, les processus de supervision et les compétences technologiques, mais ceux-ci ne relèvent pas uniquement de la distance, mais d'une utilisation des TIC en formation pratique.

Or, que la supervision de stage se fasse à distance ou en présentiel, la collaboration entre les différents accompagnateurs passe de plus en plus par les technologies, d'où notre intérêt pour un programme comme la MQES qui témoigne d'un partenariat atypique d'une université avec ses partenaires du milieu scolaire. Des entretiens auprès de superviseurs de la MQES ont permis d'identifier quelques caractéristiques positives d'une collaboration entre superviseurs, mentors et directions d'écoles, mais également certaines faiblesses d'un dispositif (celui en place lors de l'étude de cas) qui permet tout de même aux superviseurs d'être présents pour leurs stagiaires malgré la distance.

Afin de miser sur les TIC pour ainsi mieux inclure (et former) les différents partenaires, nous avons proposé une posture souhaitable en supervision de stage à distance, ainsi qu'une représentation du collectif dans lequel cette posture peut être mise à contribution. Tout cela doit se faire au service du développement professionnel du stagiaire. Même en ajoutant les pairs et les autres professionnels de l'école à la tétrade, c'est bel et bien le stagiaire qui demeure le principal acteur de sa formation. 


\section{Bibliographie}

AKYOL, Z. et GARRISON, D. R. (2011). Understanding cognitive presence in an online and blended community of inquiry: assessing outcomes and processes for deep approaches to learning. British journal of educational technology, 42(2), 233-250.

ALGER, C. ET KOPCHA, T. J. (2009). eSupervision: a technology framework for the 21st century field experience in teacher education. Teacher education, 18(2), 31-46.

ALGER, C. ET KOPCHA, T. (2011). Technology supported cognitive apprenticeship transforms the student teaching field experience: improving the student teaching field experience for all triad members. The teacher educator, 46(1), 7188.

ANDREW, L. (2007). A New University Supervisor : Their Experiences and Enrichment. Essays in Education, 22, $12-27$.

ARBAUGH, J. B. (2010). Sage, guide, both, or even more? An examination of instructor activity in online MBA courses. Computers and education, 55(3), 1234-1244.

AWAYA, A., MC EWAN, H., HEYLER, D., LINSKY, S., LUM, D. ET WAKUKAWA, P. (2003). Mentoring as a journey. Teaching and teacher education, 19, 45-56.

BAECHER, L., MCCORMACK, B. ET KUNG, S.-C. (2014). Supervisor use of video as a tool in teacher reflection. The electronic journal for english as a second language, 18(3), 1-16.

BOUDREAU, P. (2009). Pour un modèle de supervision de type inductif en formation à la supervision de maîtres de stage en éducation physique. Éducation et francophonie, 37(1), 121-139.

BOUTET, M. ET ROUSSEAU, N. (2002). Les enjeux de la supervision pédagogique des stages. Québec : Presses de I'Université du Québec.

CARAGUEL, V. (2012). E-learning et émancipation : technologie versus tutorat humain, quels facteurs d'autonomisation? In Muriel Briançon (dir.), De l'émancipation par la relation en présence à l'émancipation par les interactions à distance : quelle(s) différence(s)? Actes du Colloque Rennes2-CREAD « Formes d'éducation et processus d'émancipation », 22 mai.

CARLISLE, R. M., CARLISLE, K. L., HILL, T., KIRK-JENKINS, A. J. ET POLYCHRONOPOULOS, G. B. (2013). Distance supervision in human services. Journal of human services, 33(1), 17-28.

CHIPCHASE, L., HILL, A., DUNWOODIE, R., ALLEN, S., KANE, Y., PIPER, K. ET RUSSELL, T. (2014). Evaluating telesupervision as a support for clinical learning: an action research project. International journal of practice-based learning in health and social care, 2(2), 40-53.

CICCO, G. (2014). Building effective supervisory relationships in the online counseling course: faculty and student responsibilities. I-manager's journal on school educational technology, 10(2), 1-8.

CONN, S. R., ROBERTS, R. L. ET POWELL, B. M. (2009). Attitudes and satisfaction with a hybrid model of counselling supervision. Educational technology \& society, 12(2), 298-306.

DEAUDELIN, C., PETIT, M. ET BROUILLETTE, L. (2016). Assurer la présence enseignante en formation à distance: des résultats de recherche pour guider la pratique en enseignement supérieur. Tréma, 44(1), 79-100.

FERRIER-KERR, J. L. (2009). Establishing professional relationships in practicum settings. Teaching and teacher education, 25(6), 790-797.

GARRISON, D. R., ANDERSON, T. ET ARCHER, W. (2000). Critical inquiry in a text-based environment: computer conferencing in higher education. Internet and higher education, 2, 87-105.

GARRISON, D. R., CLEVELAND-INNES, M. ET SHING FUNG, T. (2010). Exploring causal relationships among teaching, cognitive and social presence: student perceptions of the community of inquiry framework. Internet and higher education, 13, 31-36.

GRABLE, C., HUNT, A. ET UNRUE, E. (2008). Comparing digital to traditional field experience in pre-service teacher education. In K. McFerrin et al. (dir.), proceedings of Society for information technology \& teacher education international conference (p. 400-405). Chesapeake, VA: AACE.

GRONN, D., ROMEO, G., MCNAMARA, S. ET TEO, Y.H. (2013). Web conferencing of pre-service teachers' practicum in remote schools. Journal of technology and teacher education, 21(2), 247-271. 
HAMEL, C. (2012). Supervision of pre-service teacher: using Internet collaborative tools to support their return to their region of origin. Canadian journal of education, 35(2), 141-154.

HASTINGS, W. ET SQUIRES, D. (2002). Restructuring and reculturing: practicum supervision as professional development for teachers. Asia-Pacific journal of teacher education, 30(1), 79-91.

HENRI, F. ET LUNDGREN-CAYROL, K. (2001). Apprentissage collaboratif à distance. Pour comprendre et concevoir les environnements d'apprentissage virtuels. Québec : Presses de l'Université du Québec.

HOSLER, K. A. ET AREND, B. D. (2012). The importance of course design, feedback, and facilitation : Student perceptions of the relationship between teaching presence and cognitive presence. Educational media international, 49(3), 217-229.

JÉZÉGOU, A. (2010). Créer de la présence à distance en e-learning. Cadre théorique, définition, et dimensions clés. Distances et savoirs, 8, 257-274.

JIANG, M., PARENT, S. ET EASTMOND, D. (2006). Effectiveness of web-based learning opportunities in a competencybased program. International journal on e-learning, 5(3), 353-361.

JOSEPH, G. E. ET BRENNAN, C. (2013). Framing quality: annotated video-based portfolios of classroom practice by preservice teachers. Early childhood education journal, 41, 423-430.

KOZAN, K. ET RICHARDSON, J.C. (2014). Interrelationships between and among social, teaching, and cognitive presence. Internet and higher education, 21, 68-73.

LE CORNU, R. ET EWING, R. (2008). Reconceptualising professional experiences in pre-service teacher education: reconstructing the past to embrace the future. Teaching and Teacher Education, 24, 1799-1812.

LEHMAN, R. M. ET CONCEIÇÃO, S. C. O. (2010). Creating a sense of presence in online teaching. San Francisco : Jossey-Bass.

LOUGHRAN, J.J. (2002) Effective reflective practice: in search of meaning in learning about teaching. Journal of teacher education, 53(1), 33-43.

MABUNDA, P. L. (2013). Towards a theoretical framework for the use of ICT strategies for teaching practicum supervision. Africa education review, 10(sup1), S7-S27.

MINISTĖRE DE L'ÉDUCATION, DU LOISIR ET DU SPORT (MÉLS) (2008). La formation à l'enseignement. Les orientations relatives à la formation en milieu de pratique, Québec.

MOUSSAY, S. (2007). Développer son expérience professionnelle dans le métier d'enseignant : la multiplicité de situations tutorales en formation initiale. Actes du colloque CERFEE et LIRDEF-IUFM Montpellier Compétences et socialisation, Montpellier.

MOUSSAY, S., ÉTIENNE, R. ET MÉARD, J. (2009). Le tutorat en formation initiale des enseignants : orientations récentes et perspectives méthodologiques. Revue française de pédagogie, 166.

NAULT, T. ET G. NAULT (2001). Quand les stages attrapent les TIC. In Karsenti (dir.), Les TIC... au coeur des pédagogies universitaires (pp.145-164). Québec : Presses de l'Université du Québec.

ORR, P. P. (2010). Distance supervision : research, findings, and considerations for art therapy. The arts in psychotherapy, 37(2), 106-111.

PELLERIN, G. (2010). Une étude descriptive d'un modèle de supervision en distanciel faisant appel aux TIC lors des stages réalisés dans les milieux scolaires éloignés de leur université. Thèse de doctorat, Université de Montréal, Québec.

PERAYA, D. (2015). Entre distance et présence, quelle place pour les enseignants ? Communication présentée au colloque international en éducation du CRIFPE, Montréal, 30 avril.

PETIT, M. (2015). Formation à distance et stages en enseignement : considérations pédagogiques, organisationnelles, technologiques et éthiques. In L. Thomas et M. Hirschkorn (dir.), Change and progress in Canadian teacher education: research on recent innovations in teacher preparation in Canada (p. 254-284). ACFE.

PETIT, M. (2016). Caractéristiques d'une supervision à distance de stagiaires en enseignement en ce qui concerne la création d'un sentiment de présence. Revue internationale de pédagogie de l'enseignement supérieur (RIPES), 32(1).

PETIT, M., DIONNE, L. ET BROUILLETTE, L. (2017). Remote internship supervision: state of practices in different fields of post-secondary training. Communication présentée au 10th International Conference on e-Learning \& Innovative Pedagogies, Toronto, Canada, 27 mai. 
PORTELANCE, L., GERVAIS, C., LESSARD, M. ET BEAULIEU, P. (2008). La formation des enseignants associés et des superviseurs universitaires. Rapport de recherche. Cadre de référence. Québec : Ministère de l'Éducation, du Loisir et du Sport.

RODGERS, A. ET KEIL, V.L. (2007). Restructuring a traditional student teacher supervision model: fostering enhanced professional development and mentoring within a professional development school context. Teaching and teacher education, 23, 63-80.

ROUSMANIERE, T., ABBASS, A. ET FREDERICKSON, J. (2014). New developments in technology-assisted supervision and training: a practical overview. Journal of clinical psychology, 70(11), 1082-1093.

SCHWARTZ-BECHET, B. (2014). Virtual supervision of teacher candidates: a case study. The international journal of learning: annual review, 21.

ST-ARNAUD, Y. (2003). L'interaction professionnelle : efficacité et coopération. Montréal : Presses de l'Université de Montréal.

TINTO, V. (2003). Learning better together: the impact of learning communities on student success. Higher education monograph series, 1(8).

TRAVER, A.E., VOLCHOK, E., BIDJERANO, T. ET SHEA, P. (2014). Correlating community college students' perceptions of community of inquiry presences with their completion of blended courses. Internet and higher education, 20, 1-9.

VAZQUEZ, E. ET SEVILLANO, L. (2013). ICT strategies and tools for the improvement of instructional supervision: the virtual supervision. The Turkish online journal of educational technology, 12(1), 77-87.

WOLF, N. (2003). Learning to teach mathematics for understanding in the company of mentors. Teachers and teaching theory and practice, 9(2), 87-106.

YEH, C. J., CHANG, T., CHIANG, L., DROST, C. M., SPELLISCY, D., CARTER, R. T. ET CHANG, Y. (2008).

Development, content, process and outcome of an online peer supervision group for counselo trainees. Computers in Human Behavior, 24, 2889-2903. 